\title{
On the reliability of examining dual-tasking abilities using a novel e-Health device - a proof of concept study in Multiple Sclerosis
}

\author{
Niels Böttrich ${ }^{1, \#}$, Moritz Mückschel ${ }^{1,2, \#}$, Anja Dillenseger ${ }^{1}$, Christoph Lange $^{3}$, Raimar Kern $^{3, \#}$, Tjalf $^{\prime}$ \\ Ziemssen $^{1, \#}$ and Christian Beste ${ }^{2, \#, *}$ \\ 1 MS Center Dresden, Centre for Clinical Neuroscience, Department of Neurology, Faculty of Medicine, TU Dresden, \\ Germany; niels.boettrich@gmail.com; moritz.mueckschel@ukdd.de; tjalf.ziemssen@ukdd.de \\ 2 Cognitive Neurophysiology, Department of Child and Adolescent Psychiatry, Faculty of Medicine, TU Dresden, \\ Germany; moritz.mueckschel@ukdd.de; christian.beste@ukdd.de \\ 3 MedicalSyn GmbH, Germany; raimar.kern@medicalsyn.com; christoph.lange@medicalsyn.com
}

\section{\# These authors contributed equally}

\begin{abstract}
The assessment of neuropsychological functions and especially dual-tasking abilities is considered to be increasingly relevant in the assessment of neurological disease and Multiple Sclerosis (MS) in particular. Yet, the assessment of dual-tasking abilities is hindered by specific software requirements and extensive testing times. We designed a novel e-health (progressive web application-based) device for the assessment of dual-tasking abilities usable in "bedside" and outpatient clinic settings and examined its reliability in a sample of $\mathrm{N}=184$ MS patients in an outpatient setting. Moreover, we examined the relevance of dual-tasking assessment using this device with respect to clinically relevant parameters in MS. We show that a meaningful assessment of dual-tasking is possible within 6 minutes and that reliabilities of the behavioral readouts ranged between .81 to .92 depending on dual-tasking difficulty. We show that dual-tasking readouts were correlated with clinically relevant parameters (e.g. EDSS, disease duration, processing speed) and were not affected by fatigue levels. We consider the tested dual-tasking assessment device suitable for routine clinical neuropsychological assessments of dual-tasking abilities. Future studies may further evaluate this test regarding its suitability in the long-term follow up assessments and to assess dual-tasking abilities in other neurological and psychiatric disorders.
\end{abstract}

Keywords: multiple sclerosis; neuropsychology; assessment; dual-tasking; cognition; e-health; progressive web application

\section{Introduction}

The assessment of cognitive dysfunctions in Multiple Sclerosis (MS) is central to the characterization and staging of the disease $(1-3,3,4)$ which leads to defining specific MS phenotypes (5). Several cognitive screening batteries have been developed. However, traditionally, the assessment of cognitive functions in MS is most centered around the examination of processing speed, attentional problems and memory problems, because these domains are among the first to show deficits in MS $(3,6,7)$. However, it has been argued that most of these neuropsychological assessments are limited, because they do no tap into abilities often referred to as "multitasking" (4); i.e. being able to cope with at least two demands at virtually the same time. Particularly given that these abilities are important to cope with everyday life demands and other occupational requirements a reliable assessment of these ability is highly desirable (4), and thus represent a largely unmet medical need for a holistic treatment of MS-patients. MS can be understood as a dysfunction of the human connectome (8) due to lesions in white matter structures (9-11). Since large-scale networks have been shown to be important in dual-tasking (12-20) it seems reasonable that the assessment of dual-tasking abilities is essential in the neuropsychological assessment of MS. Yet, in the field of MS- 
research, dual-tasking has mostly been examined assessing the performance of simple cognitive tasks while walking $(21-26)$ or balancing $(27,28)$. These approaches, focusing on cognition-motor interactions (26), however, bear the problem that task difficulty is not parametrically scalable and are thus possibly be unsuitable to detect early dysfunctions and track disease progression (29). Moreover, the assessment is not well controllable.

To overcome these issues, a previous study by our group introduced the usage of dual-task to examine above-mentioned abilities in MS patients (29). This test is based on a so-called psychological refractory period (PRP) task. The PRP is a well-known phenomenon (30) and describes the finding that responses (RT2) on an external signal (stimulus) of a second task (S2) are slower or less accurate when this stimulus input is presented shortly after another (first) stimulus (S1) signalling a different reaction (RT1) (=PRP effect). Varying the time between $\mathrm{S} 1$ and S2 it is possible to scale the magnitude of the PRP effect and the difficulty of the task. With increasing time between S1 and S2, the PRP effect becomes smaller (31-33), because response selection processes become less taxed (34-36). We have shown (29) that MS patients performed considerably worse than healthy control participants and that deficits shown by the patients are very likely not due to simple motor deficits. Crucially, until now, the assessment of dual-tasking abilities using the PRP-test was time consuming since test administration took about 30 minutes. More important, however, the administration of the test was difficult in outpatient settings because it required specific software packages and standard desktop PCs. Clinical usage and dissemination are strongly expedited if the test is short and can, ideally, be delivered flexibly - i.e. without specific software requirements in various settings. Therefore, the current study presents an e-health device allowing a PRP-based dual-tasking assessment using a tablet computer-based application which is already used in clinical practice (37). We present data from a sample of N 200 MS patients and examine the reliability of dual-task assessment in this patient group, as well as the relation of dual-tasking performance using this tablet computer with clinically relevant parameters in MS.

\section{Materials and Methods}

Patient sample description

In total, N=206 MS patients were prospectively recruited at the MS Centre Dresden, University Hospital Carl Gustav Carus, Germany. The testings took place within the typical outpatient setting of the MS Centre Dresden. The study was approved by the local ethics committee. Written informed consent was obtained from all participants in accordance with the Declaration of Helsinki. The ethics committee of the TU Dresden approved the study.

Due to incomplete patient data regarding disease activity, and upper extremity motor disabilities $\mathrm{N}=22$ patients were removed from the sample before data analysis was conducted. $\mathrm{N}=184$ patients $(\mathrm{N}=130$ female and $\mathrm{N}=54$ male) were included in the final analysis. The participants received no financial reimbursement for their participation. Participants were advised that participation but also non-participation in the study would not have any beneficial or detrimental effects on their patient care. The mean age was $42.04( \pm 10.8) . \mathrm{N}=78$ patients had an average premorbid intellectual performance level (as estimated by educational and professional requirements level), $\mathrm{N}=90$ were above average and $\mathrm{N}=10$ below average. $\mathrm{N}=175$ participants were relapsing-remitting (RR) MS patients ( $\mathrm{N}=68$ active, $\mathrm{N}=95$ non-active), $\mathrm{N}=5$ secondary progressive (SP) MS and $\mathrm{N}=4$ primary progressive (PP) MS patients. For $\mathrm{N}=171$ patients no disease activity (i.e. worsening of symptoms, relapses, MRI activity) was reported within the last 6 months. Medication was Ocrelizumab ( $N=48)$, Dimethylfumarate $(\mathrm{N}=30)$, Fingolimod $(\mathrm{N}=25)$, Natalizumab $(\mathrm{N}=19)$, Alemtuzumab $(\mathrm{N}=16)$, Glatiramer Acetate $(N=9)$, Teriflunomide $(N=9)$, Other $(N=24)$, None $(N=4)$. The average time since diagnosis was $10.5( \pm 7.3)$ years. All patients completed the Multiple Sclerosis Performance Test (MSPT), a tablet computer based disability assessment tool (38) including the quality of life assessment Neuro-QOL (39) within 3 months before study participation as part of standard MSPATHS clinical routine (40). The Expanded Disability Status Scale (EDSS; 41) and Multiple 
Sclerosis Severity Score (MSSS; 42) was assessed by a trained physician within the last three months prior to the study participation. The MSPT, EDSS and MSSS scores are given in Table 1.

Table 1. EDSS, MSSS and MSPT scores. The mean and standard deviation (SD) are given.

\begin{tabular}{|c|c|c|}
\hline & mean & SD \\
\hline \multicolumn{3}{|l|}{ EDSS } \\
\hline Total score & 2,5 & 1,4 \\
\hline Visual & 0,6 & 0,8 \\
\hline Brainstem & 0,6 & 0,6 \\
\hline Cerebellar & 0,9 & 0,9 \\
\hline Sensory & 1,1 & 1,0 \\
\hline Bowel_Bladder & 0,5 & 0,8 \\
\hline Cerebral & 1,0 & 0,9 \\
\hline Ambulation & 0,8 & 1,8 \\
\hline \multicolumn{3}{|l|}{ MSSS } \\
\hline Total score & 3,2 & 2,0 \\
\hline \multicolumn{3}{|l|}{ MSPT } \\
\hline Processing Speed Test / SDMT & 56,5 & 13,5 \\
\hline Low Contrast Letter Acuity Test & 39,7 & 8,0 \\
\hline Manual Dexterity Test right & 22,6 & 6,2 \\
\hline Manual Dexterity Test left & 22,7 & 5,8 \\
\hline Walking Speed Test & 5,2 & 2,2 \\
\hline \multicolumn{3}{|l|}{ Neuro-QoL } \\
\hline Ability to Participate in Social Roles and Activities & 49,8 & 8,0 \\
\hline Satisfaction with Social Roles and Activities & 49,7 & 7,2 \\
\hline Depression & 44,9 & 7,9 \\
\hline Emotional and Behavioral Dyscontrol & 47,7 & 9,3 \\
\hline Stigma & 44,4 & 7,9 \\
\hline Applied Cognition & 50,7 & 9,5 \\
\hline Positive Affect and Well-Being & 102,1 & 584,9 \\
\hline Fatigue & 45,0 & 10,0 \\
\hline Sleep Disturbance & 48,2 & 10,2 \\
\hline Lower Extremity (Mobility) & 53,6 & 9,0 \\
\hline Upper Extremity (Fine Motor) & 49,5 & 8,9 \\
\hline
\end{tabular}

\section{Dual-task}

The dual-task was conducted on a Galaxy Tab A 10.5 (SM-T590) with Android 9 (Samsung Electronics Co., Ltd.) tablet computer with a $24.54 \mathrm{~cm}$ capacitive touchscreen, a screen resolution of $1920 \times 1200$ px, running on the Android (Open Handset Alliance) operating system. The dual-task was identically structured as in a previous study first introducing this test in the cognitive assessment of MS patients (29). The structure of the task (i.e. succession of stimuli and required responses) is shown in Figure 1. There was a "tone task" and a "letter task" (43). Tones were delivered via headphones and were pitches of $300 \mathrm{~Hz}$ or $900 \mathrm{~Hz}$ frequency. Each tone was presented for $200 \mathrm{~ms}$. During the "letter task", the letters " $\mathrm{H}$ " and " $\mathrm{O}$ " were used to ease discrimination of the stimuli. The dimension of the stimuli were $1.2 \times 1 \mathrm{~cm}$ and the stimuli were presented in the centre of the screen. 

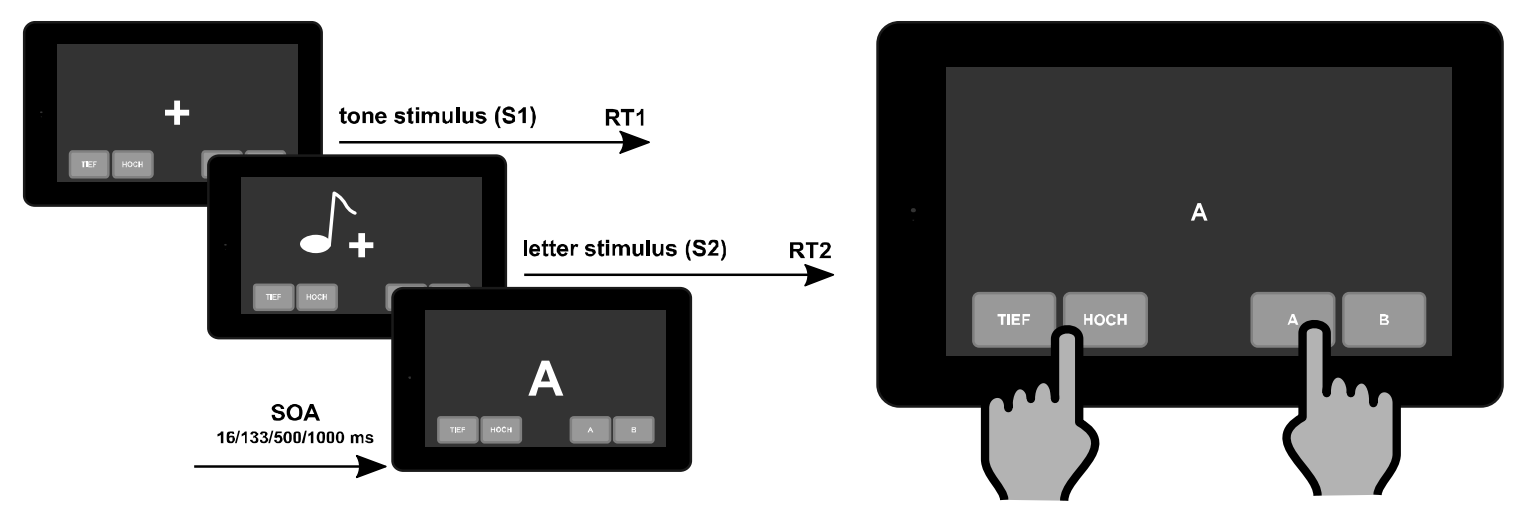

Figure 1. Schematical illustration of the PRP paradigm. The tone stimulus (S1) is presented first. The letter stimulus (S2) is presented with a defined stimulus-onset asynchrony (SOA). Participants are asked to respond as soon as possible to the tone stimulus by pressing one of two buttons with their left index finger and to respond as fast as possible to the letter stimulus by pressing one of two buttons with their right index finger.

The tone task was always presented first. The time differences between the presentation of the tone stimulus and the presentation of the letter stimulus (stimulus onset asynchrony, SOA) was varied in 4 steps: $16 \mathrm{~ms}, 133 \mathrm{~ms}, 500 \mathrm{~ms}$ and $1000 \mathrm{~ms}$. Each SOA was presented 36 times. The test was divided into 3 blocks of 48 trials each. In each block, all possible tone-letter combinations and the different SOAs occurred equally frequent and were presented in a pseudo-randomized fashion. To respond in the task, the participants had to operate four buttons. Two of these were located in the left corner (one for a high pitch and one for a low pitch), the two in the right corner of the tablet's touchscreen (one for letter " $A$ " and one for letter "B"). A left-hand index finger response was performed for tones, a right index finger response for the letters. Each trial started with the presentation of a central fixation cross. The response time window was restricted to $2000 \mathrm{~ms}$. If no response occurred within this period, the trial was considered a miss. In this case, the next trial started within a randomly jittered interval of 500 to $2500 \mathrm{~ms}$ (mean $1500 \mathrm{~ms}$ ). If a valid response was given, the next trial started after a response stimulus interval (RSI) of 2000 ms, jittered between 1000 and 4000 ms. Participants were asked to respond as quickly and accurately as possible and to place equal emphasis on both tasks. Additionally, the participants were instructed to respond first on the tone stimulus (S1) and second on the letter stimulus (S2). Prior to the experiment, all participants completed 2 exercise blocks of each 12 trials.

Implementation of the dual-task as mobile e-health tool

The Dual-Task, described above, was developed as a server-based progressive web application. Therefore, it can be executed on a wide range of devices, like tablet computers, smartphones etc. Dual-tasking based patient testing is carried out in the web browser and the data is stored and processed on the corresponding server. The application is divided into front-end and back-end. The front-end part runs in the browser of the end user on the end user's mobile device. In the Vue.jsbased interface of the front-end, the stimuli used in the task are presented. The participant can learn the functions of the interface in a tutorial. The front-end is optimized in such a way that no disturbing influences from the user interface are displayed and the participant can concentrate completely on the execution of the dual-tasking test. After completion of a test, the participant can view and evaluate the test results in a detailed table view. The front-end is connected to the back-end via a secure HTTP over TLS connection using state-of-the-art REST interfaces. This means that all data transfer between front-end and back-end is encrypted and therefore all test results are encrypted and securely transmitted. The back-end is based on ASP.Net Core 3.1. ASP.NET is a modern and popular web-development framework for building web apps and the NET. platform and use in all 
common server operating systems. The back-end securely and efficiently stores test results on the web server for easy retrieval, post-processing or evaluation.

\section{Statistical analyses}

Statistical analysis was conducted using Matlab 2019a (The MathWorks, Inc.) and SPSS (IBM Corp.). For analysis of the PRP effect, the slope of the SOA function was computed for letter stimulus RTs

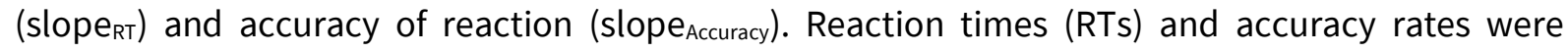
analyzed using repeated measures ANOVAs with the within-subject factor "SOA" (SOA 16 vs. SOA 133 vs. SOA 500 vs. SOA 1000). ANOVAs were conducted for the overall results, separately for block 1 to 3 and for the data of block 1 and 2 as well as block 2 and 3. Greenhouse-Geisser corrections were applied when necessary. Pair-wise comparisons are Bonferroni-corrected. For correlation analysis, Pearson's linear correlation coefficient was computed. For reliability analysis, Cronbach's alpha was computed for block 1, 2 and 3.

\section{Results}

Due to the focus on the PRP effect (i.e. the modulation of response selection processes triggered by the second stimulus), the analysis was limited to the RTs and the accuracy rates to the letter stimulus (S2). Mean RTs and hit rates for each block are given in Figure 2. Mean RTs, hit rates, slope ${ }_{\text {RT }}$ and slope $_{\text {Accuracy }}$ function per block are given in Table 2 .
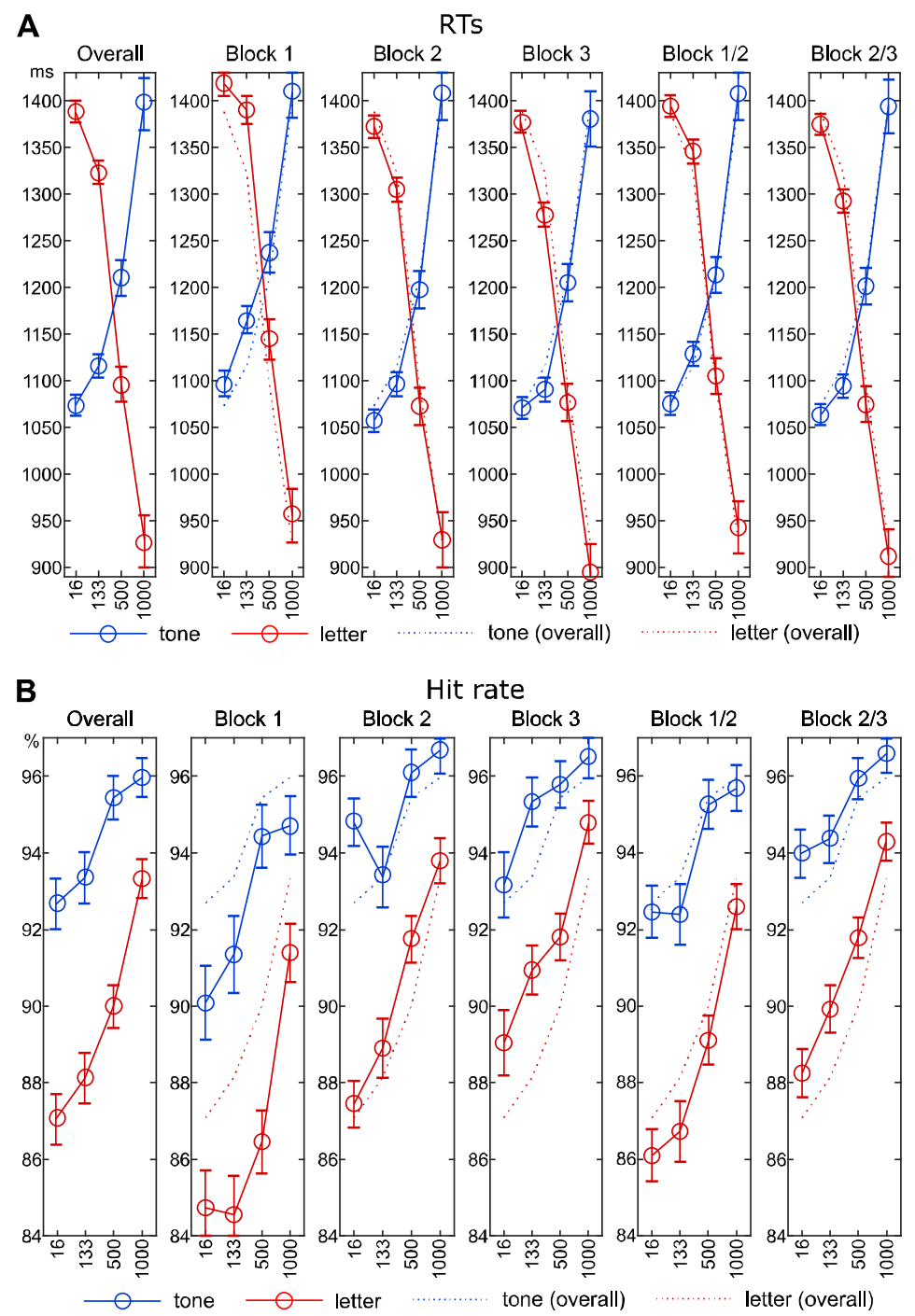
Figure 2. RTs (A) and hit rates (B) for the tone and letter stimulus, separately for the entire test (overall), for each block, aggregated for block 1 and 2 as well as for block 2 and 3 . The $x$-axis denotes the SOA, the $y$-axis denotes the RT in ms (A) or accuracy rate in \% (B). Error bars indicate standard error of the mean (SEM). The line color denotes tone stimulus (blue) or letter stimulus (red). The dashed line indicates data from overall blocks for comparison.

Table 2. Mean RTs, accuracy rates, slope RT and $_{\text {Slope }}$ Accuracy $( \pm$ standard error of the mean, SEM) of the letter stimulus (S2) for each SOA condition.

\begin{tabular}{cccccc}
\hline & SOA 16 & SOA 133 & SOA 500 & SOA 1000 & Slope SOA \\
\hline S2 RTS & & & & & \\
Overall & $1388.5 \pm 14$ & $1322.9 \pm 14.5$ & $1095.4 \pm 18.9$ & $926.5 \pm 19.1$ & $462 \pm 11.2$ \\
block 1 & $1419 \pm 14.8$ & $139.5 \pm 16.9$ & $1145.3 \pm 21$ & $957.2 \pm 2.5$ & $461.9 \pm 12.9$ \\
block 2 & $1372.5 \pm 15.3$ & $1304.9 \pm 15.7$ & $1072.9 \pm 19.7$ & $929.5 \pm 2.4$ & $443 \pm 12.5$ \\
block 3 & $1377.3 \pm 14.5$ & $1277.7 \pm 14.4$ & $1076.5 \pm 19.7$ & $894.9 \pm 19$ & $482.3 \pm 13.3$ \\
block 1/2 & $1394.3 \pm 14.4$ & $1346 \pm 15.4$ & $1105.2 \pm 19.2$ & $942.9 \pm 19.8$ & $451.5 \pm 11.6$ \\
block 2/3 & $1375 \pm 14.4$ & $1292.6 \pm 14.4$ & $1074.6 \pm 19$ & $912 \pm 19.2$ & $463 \pm 11.8$ \\
S2 accuracy & & & & & \\
Overall & $87.1 \pm 1.2$ & $88.1 \pm 1$ & $90 \pm .9$ & $93.3 \pm .7$ & $-6.3 \pm .8$ \\
block 1 & $84.7 \pm 1.3$ & $84.6 \pm 1.3$ & $86.5 \pm 1.2$ & $91.4 \pm .9$ & $-6.7 \pm 1.1$ \\
block 2 & $87.5 \pm 1.3$ & $88.9 \pm 1.1$ & $91.8 \pm .9$ & $93.8 \pm .9$ & $-6.3 \pm 1.2$ \\
block 3 & $89 \pm 1.2$ & $9.9 \pm 1.1$ & $91.8 \pm .8$ & $94.8 \pm .8$ & $-5.8 \pm .9$ \\
block 1/2 & $86.1 \pm 1.2$ & $86.7 \pm 1.1$ & $89.1 \pm 1$ & $92.6 \pm .8$ & $-6.5 \pm 1$ \\
block 2/3 & $88.2 \pm 1.2$ & $89.9 \pm 1$ & $91.8 \pm .8$ & $94.3 \pm .8$ & $-6 \pm .9$ \\
\hline
\end{tabular}

\section{Reaction times}

Regarding the RTs to the letter stimulus in correct trials for all blocks i.e. all trials, the repeated measures ANOVA showed a significant main effect of SOA $\left(F(1.69,309.68)=1193.04 ; p<.001 ; \eta^{2} p=\right.$ .87). Bonferroni-corrected pairwise comparisons showed that the RTs of all SOA differed significantly from each other (all $p<.001$ ) and significantly decreased from SOA 16 to SOA 1000.

Looking at block 1 (i.e. the initial first third of all trials), the ANOVA for RTs again yielded a significant main effect $\left(F(2.34,427.28)=715.46 ; p<.001 ; \eta^{2} p=.8\right)$. Similar to the overall analysis, RTs decreased with increased SOA and all SOA differed significantly from each other $(p<.002)$.

For block 2 there was a significant main effect $\left(F(2.011,367.999)=757.2 ; p<.001 ; \eta^{2} p=.81\right)$. RTs of all SOA differed significantly from each other $(p<.001)$ and decreased with longer SOA. A main effect was also found for hit rates $\left(F(2.63,480.5)=16.56 ; p<.001 ; \eta^{2} p=.08\right)$.

Block 3 RTs again showed a main effect $\left(F(2.22,405.76)=712,46 ; p<.001 ; \eta^{2} p=.8\right)$. RTs decreased with longer SOA and all SOA RTs differed significantly $(p<.001)$.

For the RTs of block 1 and 2, i.e. the first two third of trials) the repeated measures ANOVA showed a main effect $\left(F(1.82,332.26)=1052.06 ; p<.001 ; \eta^{2} p=.85\right)$. The RTs differed between all blocks $(p<$ .001 ) and longer SOA were linked to shorter RTs.

Regarding trials of block 2 and 3, i.e. the last two third of trials, a main effect was observed $\left(F(1.79,326.9)=1003.67 ; p<.001 ; \eta^{2} p=.85\right)$. RTs decreased with larger SOA and RTs differed between all SOA $(p<.001)$.

\section{Accuracy data}

Regarding the accuracy (hit rate) to the letter stimulus in correct trials for all blocks i.e. all trials, the ANOVA showed a significant main effect $\left(F(2.28,416.35)=36.66 ; p<.001 ; \eta^{2} p=.17\right)$. The number of 
hits differed significantly between all SOA ( $p<.004)$, except for SOA 16 and SOA 133. Longer SOA were connected to higher hit rates.

In block 1, For hit rates the ANOVA also showed a main effect $\left(F(2.82 ; 515.42)=21.25 ; p<.001 ; \eta^{2} p=\right.$ .1). Hit rates at SOA 1000 differed significantly from all other SOA $(p<.001)$, but no significant differences were found between SOA 16, SOA 133 and SOA 500 ( $p>.229)$.

For block 2, hit rates of the different SOAs differed significantly $(p<.007)$ except for SOA 16 and SOA $133(p=.765)$ as well as $S 500$ and $S 1000(p=.099)$. Longer SOAs were associated with higher hit rates.

In block 3, the accuracy also differed significantly as shown by a significant main effect $(F(2.67$; 488.17) $\left.=19.45 ; p<.001 ; \eta^{2} p=.1\right)$. Longer SOAs were connected with higher hit rates $(p<.007)$ but no differences were found for SOA 16 and SOA $133(p=.055)$ and SOA 133 and SOA $500(p=1)$.

For the accuracy pooled across blocks 1 and 2, a significant main effected could also be found $\left(F(2.41,441.53)=28.05 ; p<.001 ; \eta^{2} p=.13\right)$. Significant differences were evident between all SOA $(p<$ $.006)$ except for SOA16 and SOA133 $(p=1)$ and longer SOAs were associated with increased hit rates. Regarding trials of block 2 and 3, i.e. the last two third of trials, the main effect was also significant $\left(F(2.3,421.48)=28.1 ; p<.001 ; \eta^{2} p=.13\right)$. Except for SOA 16 and SOA $133(p=.054)$, hit rated differed significantly between SOA $(p<.007)$ and longer SOAs were connected to larger hit rates.

\section{Reliability analysis}

For reliability analysis, Cronbach's a was calculated for RTs and the accuracy data, as well as the slope $_{R T}$ and the slope Accuracy $_{\text {to }}$ assess the internal consistency of blocks 1 to 3 (see Table 3 for the RT data and Table 4 for the accuracy data). This was done separately for the tone stimulus (S1) and the letter stimulus (S2). However, the most important parameters are these in response to the letter stimulus (S2), since these reflect SOA-dependent modulations in response selection (i.e. dualtasking abilities and the PRP effect). As can be seen in Tables 3 and 4, the internal consistency is satisfying with Cronbachs alpha raging between .92 and .94 for the reaction time data depending on the SOA condition. Also for the accuracy data, Cronbachs alpha was reasonable with ranges between .81 and .89 depending on SOA condition. For the slope of the RT and accuracy parameters Cronbachs alpha was lower (cf. Tables 3 and 4). For all examined parameters (i.e. RTs, response accuracy, and the slope of these parameters), Block 2 showed the highest sensitivity.

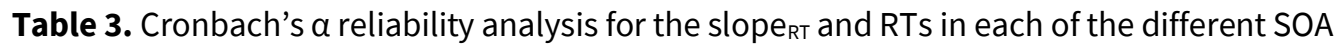
conditions and blocks

\begin{tabular}{lcccc}
\hline & $\begin{array}{c}\text { Cronbach's } \\
\text { Tone stimulus }\end{array}$ & Sensitivity & $\begin{array}{c}\alpha \text { if } \\
\text { deleted }\end{array}$ & $\begin{array}{c}\text { part- } \\
\text { whole- } \\
\text { correction }\end{array}$ \\
(S1) & & &
\end{tabular}

\begin{tabular}{lcccc}
\hline & Cronbach's & Sensitivity & $\begin{array}{c}\text { a if } \\
\text { deleted }\end{array}$ & $\begin{array}{c}\text { part- } \\
\text { whole- } \\
\text { Letter }\end{array}$ \\
stimulus (S2) & & & & correction
\end{tabular}
(S1)

\begin{tabular}{ccccc}
\hline $\begin{array}{c}\text { slope } \\
\text { block 1 }\end{array}$ & & & & \\
block 2 & .93 & .89 & .93 & -.01 \\
block 3 & & .86 & .88 & .07 \\
RTs SOA 16 & & .04 \\
block 1 & & .80 & .91 & .01 \\
block 2 & .91 & .86 & .84 & .07 \\
block 3 & & .82 & .87 & .04 \\
RTs SOA 133 & & & & \\
block 1 & & .83 & .91 & .01 \\
block 2 & .92 & .86 & .88 & .04 \\
block 3 & & .86 & .88 & .04
\end{tabular}

\begin{tabular}{ccccc}
\hline $\begin{array}{c}\text { slope } \\
\text { block 1 }\end{array}$ & & & & \\
block 2 & .84 & .64 & .84 & 0 \\
block 3 & & .71 & .79 & .06 \\
RTs SOA 16 & & & & \\
block 1 & & .85 & .93 & .01 \\
block 2 & .94 & .91 & .89 & .05 \\
block 3 & & .88 & .91 & .03 \\
RTs SOA 133 & & & & \\
block 1 & & .82 & .90 & .02 \\
block 2 & .92 & .87 & .85 & .06 \\
block 3 & & .83 & .89 & .03
\end{tabular}




\begin{tabular}{|c|c|c|c|c|c|c|c|c|c|}
\hline RTs SOA 500 & & & & & RTs SOA 500 & & & & \\
\hline block 1 & & .80 & .94 & -.01 & block 1 & & .84 & .94 & 0 \\
\hline block 2 & .93 & .89 & .86 & .07 & block 2 & .94 & .91 & .89 & .05 \\
\hline block 3 & & .86 & .89 & .04 & block 3 & & .88 & .91 & .03 \\
\hline RTs SOA 1000 & & & & & RTs SOA 1000 & & & & \\
\hline block 1 & & .88 & .96 & 0 & block 1 & & .87 & .95 & 0 \\
\hline block 2 & .96 & .94 & .92 & .04 & block 2 & .95 & .93 & .91 & .05 \\
\hline block 3 & & .92 & .93 & .02 & block 3 & & .90 & .93 & .02 \\
\hline
\end{tabular}

Table 4. Cronbach's a reliability analysis for the slope of the SOA accuracy rates function and accuracies in each of the different SOA conditions and blocks

\begin{tabular}{|c|c|c|c|c|}
\hline $\begin{array}{l}\text { Tone stimulus } \\
\text { (S1) }\end{array}$ & $\begin{array}{c}\text { Cronbach's } \\
a\end{array}$ & Sensitivity & $\begin{array}{c}\alpha \text { if } \\
\text { deleted }\end{array}$ & $\begin{array}{c}\text { part- } \\
\text { whole- } \\
\text { correction }\end{array}$ \\
\hline
\end{tabular}

\begin{tabular}{lcccc}
\hline & $\begin{array}{c}\text { Cronbach's } \\
\text { Letter }\end{array}$ & Sensitivity & $\begin{array}{c}\text { a if } \\
\text { deleted }\end{array}$ & $\begin{array}{c}\text { part- } \\
\text { whole- } \\
\text { correction }\end{array}$ \\
stimulus (S2) & & & &
\end{tabular}

$\begin{array}{ccccc}\text { slope } \text { Hit } & & & & \\ \text { block 1 } & & .20 & 0 & 0 \\ \text { block 2 } & 0 & .11 & 0 & 0 \\ \text { block 3 } & & .22 & .20 & -.20\end{array}$

\begin{tabular}{rrrrr}
\hline slope & & & & \\
block 1 & & .46 & .61 & .06 \\
block 2 & .67 & .58 & .42 & .25 \\
block 3 & & .42 & .65 & .02
\end{tabular}

Hits SOA 16

$\begin{array}{lllll}\text { block 1 } & & .57 & .61 & .11 \\ \text { block 2 } & .72 & .54 & .66 & .06 \\ \text { block 3 } & & .56 & .60 & .11\end{array}$

Hits SOA 16

Hits SOA 133

$\begin{array}{llll}\text { block } 1 & .57 & .66 & .07\end{array}$

$\begin{array}{lllll}\text { block } 2 & .73 & .63 & .55 & .18\end{array}$

block 3

$.52 \quad .70$

.03

Hits SOA 500

$\begin{array}{llll}\text { block } 1 & .58 & .70 & .05\end{array}$

$\begin{array}{lllll}\text { block } 2 & .75 & .66 & .58 & .17\end{array}$

block 3

$.53 \quad .72$

.03

Hits SOA 1000

$\begin{array}{lllll}\text { block 1 } & & .52 & .64 & .06 \\ \text { block 2 } & .70 & .59 & .53 & .17 \\ \text { block 3 } & & .48 & .66 & .04\end{array}$

$\begin{array}{lllll}\text { block 1 } & & .75 & .86 & .03 \\ \text { block 2 } & .89 & .82 & .79 & .09 \\ \text { block 3 } & & .76 & .86 & .03\end{array}$

Hits SOA 133

$\begin{array}{llll}\text { block } 1 & .72 & .83 & .03\end{array}$

$\begin{array}{lllll}\text { block } 2 & .86 & .75 & .78 & .07\end{array}$

$\begin{array}{llll}\text { block } 3 & .75 & .79 & .06\end{array}$

Hits SOA 500

$\begin{array}{lllll}\text { block 1 } & & .77 & .79 & .07 \\ \text { block 2 } & .86 & .73 & .80 & .05 \\ \text { block 3 } & & .75 & .80 & .06\end{array}$

Hits SOA 1000

\begin{tabular}{|c|c|c|c|}
\hline block 1 & & .61 & .79 \\
\hline block 2 & .81 & .70 & .70 \\
\hline block 3 & & .67 & .73 \\
\hline
\end{tabular}

\section{Correlation analysis}

The results of the correlation analysis of letter stimulus (S2) slope parameter for RTs and hit rates using MSPT, EDSS and MSSS data are given in Figure 3. These correlations were calculated to assess the clinical relevance/validity of the results. We used the slope, because this parameter provides information on strong much response selection in dual-task situations is modulated by varying degrees in difficulty (i.e. SOA).

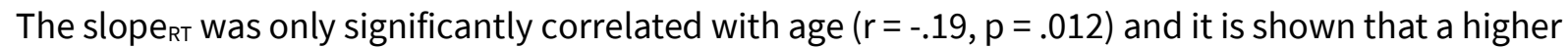
age was associated with a flatter slope ${ }_{R T}$. No correlations were found with clinical scales. However,

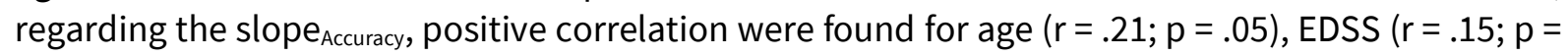


$.036)$ and disease duration $(r=.23 ; p=.02)$, indicating that increased age, higher EDSS scores and longer disease durations were connected to an increased slope $_{\text {Accuracy. }}$ In contrast, a negative correlation was found for the MSPT Processing Speed/MSPT score $(r=-.32 ; p<.01)$. Higher MSPT performance was linked to a flatter slope. Generally, no correlations were obtained for the MSSS and the Neuro-QoL Fatigue score.

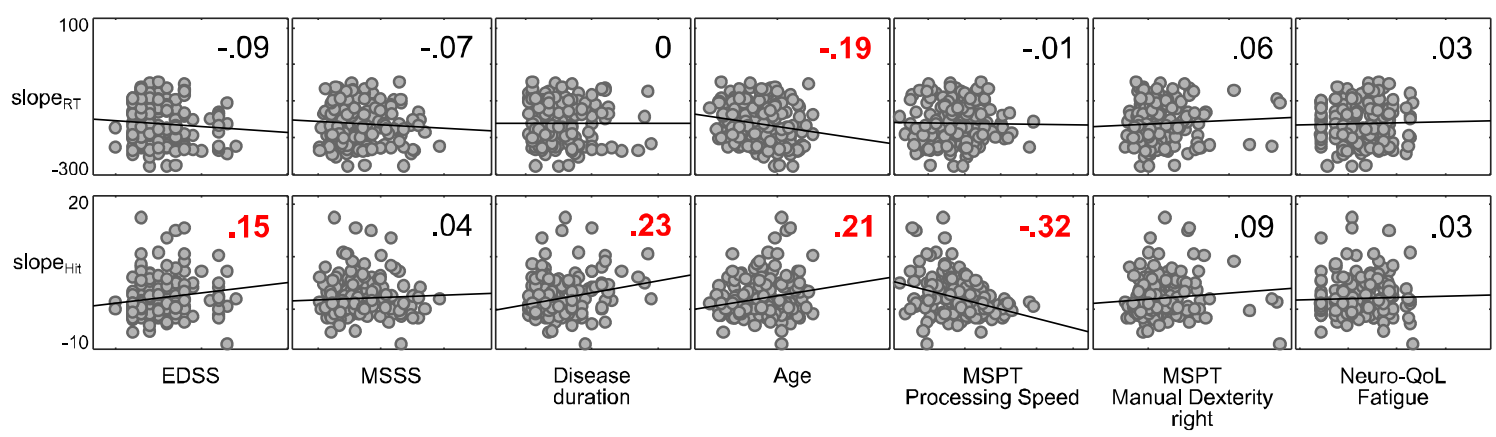

Figure 3. Results of correlation analysis. The numbers denote the correlation coefficient. Red font color indicates significant correlations $(p<.05)$.

\section{Discussion}

Goal of the study was to develop a novel progressive web application dual-tasking assessment tool (e-health device) and to examine psychometric properties of this device in terms of its reliability. Furthermore, goal of the work was to examine in how far dual-tasking abilities examined in this task are relevant to clinical MS care by examining correlations between task performance and established clinical parameters on disease severity in MS.

Previous data using the same dual-task have already shown robust differences to healthy controls (29). The current study was motivated by these findings and the problem that the assessment of dual-tasking abilities still reflects an unmet clinical need in the clinical care of MS patients (4). Until now, the problem was that dual-tasking assessment approaches derived from experimental psychology and cognitive neuroscience were not well applicable in clinical practice since test administration required specific software and was not well integratable in routine test settings (e.g. in outpatient or bedside settings) due to long testing times and bulky devices to administer the test. This problem was solved in the current study by designing a progressive web application that works on any platform.

The data reveal that a typical psychological refractory period (PRP) effects on the letter (S2) stimuli was obtained using the novel device. It is shown that reaction times were longest in the shortest SOA condition (i.e. when temporal spacing between the tone and the letter task (stimuli) was smallest). Reaction times became faster with increase SOA time (31-33). The same effect is shown for the accuracy data. Response accuracy increased from the shortest SOA condition to the longest SOA condition. Therefore, response selection accuracy in this dual task became better when the task was less taxing (34-36). This pattern of findings was evident in each block of the PRP-implementation of the device. Since one major aspect important to consider for the clinical applicability and user acceptance of the device is testing time, this study followed the approach of building three different task blocks with identical trial numbers for each SOA condition. The fact that consistent PRP effects occurred in all three blocks shows that dual-tasking abilities were measured in the same way in all three blocks. This is a major pre-requisite to design a test instrument that can be applied in short durations of time.

Most important regarding the applicability of the tablet-based implementation of the PRP is the conducted reliability analysis. As outlined in the results section, the reliability for the RT and for the accuracy parameter was reasonably high; i.e. .92 and .94 for the reaction time data depending on 
the SOA condition. Also, for the accuracy data, Cronbachs alpha was reasonable with ranges between .81 and .89 depending on SOA condition. Especially the high reliability of the accuracy data is important for the clinical applicability. The reason is that motor speed (i.e. RTs) is strongly affected in MS and can bias the applicability of motor response-dependent assessments of higher level cognitive functions. The finding that the accuracy data turned out to be reliable in the test suggests that the accuracy parameter in tablet-based PRP implementations can well be used in the neuropsychological assessment of dual-tasking abilities in MS patients. Already previous findings revealed that particularly the accuracy parameter differs between MS patients and healthy controls when examining dual-tasking performance using the PRP (29). The study by Beste et al (29) used the same setup of stimuli (tones and letters) as well as the same timing of stimuli, as the current tablet implementation of the PRP task. All this suggest that the accuracy parameter may be used to examine dual-tasking abilities in MS. The slope of the accuracy parameter revealed a smaller reliability (i.e. Cronbachs alpha) that accuracy parameters for each SOA condition separately. This is an expected (mathematical) effect, since the slope parameter represents a ratio that is associated with an increase in variance in the data. However, the data also show that particularly Block 2 showed the highest sensitivity. Each of the different three blocks in the tested implementation of the dual-task paradigm lasted 3 minutes. Therefore, the data suggest that a testing time of $\sim 6$ minutes is required and also sufficient for a reliable estimation of dual-tasking performance in MS patients. This aspect is of high practical relevance, since it fosters the applicability of dual-tasking assessments in clinical settings. Within these 6 minutes testing time, all SOA conditions were administered equally often. The variation in SOA conditions allows for "adaptive testing"(29); i.e. the test applied allows scaling the difficulty of dual-tasking by means of different SOAs. This is an important feature for the applicability of dual-tasking assessments in longitudinal studies and to track disease progression. This makes the test suitable also for adaptive testing in patients with more severe disease symptoms, especially because the accuracy parameter and not only the response speed parameter was shown to be reliable.

Considering the accuracy parameter (i.e. its slope), it is interesting that this parameter revealed correlations with clinically relevant parameters such as the EDSS score, disease duration and processing speed as assessed by MSPT. EDSS score and disease duration were positively correlated with the slope. The data suggest that response selection processes in dual-tasking become more prone to variations in the difficulty to select a response (i.e. SOA variation effect) when the EDSS score becomes higher and disease duration longer. Also processing speed, as examined using the MSPT, was correlated with the slope of the accuracy parameter. Here, a negative correlation was found suggesting that a higher processing speed was related to a flatter slope. Hence, response selection processes in dual-tasking become less prone to variations in the difficulty to select a response when the patient has a relatively high information processing speed, as examined using the MSPT. This finding is reasonable since the PRP task has strong speed component. All these results suggest that the tested dual-task implementation taps into clinically relevant aspects of the disease. However, it is important to note that correlation coefficients were low. This shows that the dual-task assessment is not redundant to existing clinically relevant measures of cognitive function such as processing speed measured using the MSPT. Rather, the data suggest that the tested ehealth device complements theses existing measures by providing a reliable assessment of dualtasking abilities in MS patients. Notably, no correlations were evident with a measure of fatigue (i.e. Neuro-QoL fatigue), which suggest that the test can also be applied when MS patients suffer from fatigue. This is central because fatigue affects other routinely used neuropsychological assessment tools $(44,45)$. The "age" of the patients was also correlated with task performance for the accuracy and the RT data, however, a positive correlation was obtained for the accuracy data and a negative correlations was obtained for the RT data. This suggest that "age" mainly induces a speed-accuracy trade-off in dual-tasking and has no other clinical meaningfulness.

The assessment of cognitive functions is becoming a cornerstone in routine clinical care and clinical trials of MS patients $(1,2,4)$. Especially with regard to the inclusion of cognitive tests in clinical 
trials, it is essential that the tests are reliable and quickly administrable. In this pilot study, we demonstrate that the test is easy to apply without intense training of nurses in clinical real-world settings. The dual-task test enables an assessment using a progressive web application, which could be applied in MS centers or by the patients themselves, which makes it quickly scalable to high case numbers in the context of clinical study situations. In addition, this clinically very relevant test (4) could be transferred to everyday clinical practice to monitor cognitive function longitudinally. We argue that especially the web-based technology of the tested device including an in-built database structure will prove useful in the clinical applicability of the device. Such e-health diagnostic tools are helpful to alleviate the supply shortfall in the healthcare system and to improve the care of chronically ill patients because they can present the course of the illness more comprehensively and more accurately than standard clinical visits, especially in MS (46). Using digital tools, data collection does not increase the burden on providers or generate significant incremental cost. So, the proliferation of computerized neuropsychological assessment devices (CNADs) for screening and monitoring cognitive impairment is increasing exponentially (47). This can support the general strategy to provide personalized MS management in which the assessment of cognitive functions using digital approaches needs to be implemented alongside immunological, genetic and MRI profiling of the individual patient $(5,48)$.

Taken together, the presented study underlines the reliability of the developed tablet-based assessment tool for dual-tasking abilities in MS patients. The results show that it is possible to conduct a reliable assessment of multitasking abilities in about 6 minutes. We consider this duration acceptable for routine clinical neuropsychological assessments of dual-tasking abilities. As such, the presented assessment tool seems suitable to address a clinical need to examine dual-tasking (4).. Future studies may also evaluate this assessment tool regarding its suitability in the long-term follow up assessments of MS patients and to assess dual-tasking abilities in other neurological and psychiatric disorders.

\section{Acknowledgements}

This work supported by a Grant from the Deutsche Forschungsgemeinschaft BE4045/10-3. We thank all participants.

\section{Author contributions}

Conceptualization, R.K., T.Z. and C.B.; methodology, M.M.; C.B.; software, C.L, R.K.; validation, M.M., N.B.; visualization, M.M ;formal analysis, N. B., M.M. and C.B.; investigation, N. B.; resources, R.K., A.D., T.Z., C.B.; data curation, R.K.; M.M; project administration, A.D., T.Z., C.B.; writing-original draft preparation, N.B., M.M., C.B.; writing-review and editing, all authors.; visualization, M.M.; supervision, T.Z.; C.B.; project administration, R.K., C.B. T.Z.; funding acquisition, C.B.. All authors have read and agreed to the published version of the manuscript.

\section{References}

1. Chiaravalloti ND, DeLuca J. Cognitive impairment in multiple sclerosis. The Lancet Neurology. 2008 Dec;7(12):1139-51.

2. DeLuca J, Chiaravalloti ND, Sandroff BM. Treatment and management of cognitive dysfunction in patients with multiple sclerosis. Nat Rev Neurol [Internet]. 2020 May 5 [cited 2020 Jun 1]; Available from: http://www.nature.com/articles/s41582-020-0355-1

3. Rocca MA, Amato MP, De Stefano N, Enzinger C, Geurts JJ, Penner I-K, et al. Clinical and imaging assessment of cognitive dysfunction in multiple sclerosis. The Lancet Neurology. 2015 Mar 1;14(3):302-17. 
4. Sumowski JF, Benedict R, Enzinger C, Filippi M, Geurts JJ, Hamalainen P, et al. Cognition in multiple sclerosis: State of the field and priorities for the future. Neurology. 2018 06;90(6):27888.

5. Ziemssen T, Kern R, Thomas K. Multiple sclerosis: clinical profiling and data collection as prerequisite for personalized medicine approach. BMC Neurol. 2016 Dec;16(1):124, s12883016-0639-7.

6. Amato MP, Langdon D, Montalban X, Benedict RHB, DeLuca J, Krupp LB, et al. Treatment of cognitive impairment in multiple sclerosis: position paper. J Neurol. 2013 Jun;260(6):1452-68.

7. Benedict RH b., Cookfair D, Gavett R, Gunther M, Munschauer F, Garg N, et al. Validity of the minimal assessment of cognitive function in multiple sclerosis (MACFIMS). Journal of the International Neuropsychological Society. 2006 Jul;12(04):549-558.

8. Griffa A, Baumann PS, Thiran J-P, Hagmann P. Structural connectomics in brain diseases. Neurolmage. 2013 Oct 15;80(Supplement C):515-26.

9. Bonzano L, Pardini M, Mancardi GL, Pizzorno M, Roccatagliata L. Structural connectivity influences brain activation during PVSAT in Multiple Sclerosis. Neurolmage. 2009 Jan $1 ; 44(1): 9-15$.

10. Bonzano L, Tacchino A, Roccatagliata L, Sormani MP, Mancardi GL, Bove M. Impairment in explicit visuomotor sequence learning is related to loss of microstructural integrity of the corpus callosum in multiple sclerosis patients with minimal disability. Neurolmage. $2011 \mathrm{Jul}$ 15;57(2):495-501.

11. Droby A, Yuen KSL, Muthuraman M, Reitz S-C, Fleischer V, Klein J, et al. Changes in brain functional connectivity patterns are driven by an individual lesion in MS: a resting-state fMRI study. Brain Imaging and Behavior. 2016 Dec 1;10(4):1117-26.

12. Chmielewski WX, Yildiz A, Beste C. The neural architecture of age-related dual-task interferences. Front Aging Neurosci [Internet]. 2014 [cited 2017 Apr 12];6. Available from: http://journal.frontiersin.org/article/10.3389/fnagi.2014.00193/abstract

13. Dux PE, Ivanoff J, Asplund CL, Marois R. Isolation of a Central Bottleneck of Information Processing with time-resolved fMRI. Neuron. 2006 Dec 21;52(6):1109-20.

14. Gohil K, Stock A-K, Beste C. The importance of sensory integration processes for action cascading. Scientific Reports. 2015 Mar 30;5:9485.

15. Gohil K, Bluschke A, Roessner V, Stock A-K, Beste C. Sensory processes modulate differences in multi-component behavior and cognitive control between childhood and adulthood. Hum Brain Mapp. 2017 Oct 1;38(10):4933-45.

16. Marois R, Larson JM, Chun MM, Shima D. Response-specific sources of dual-task interference in human pre-motor cortex. Psychol Res. 2006 Nov;70(6):436-47.

17. Stelzel C, Kraft A, Brandt SA, Schubert T. Dissociable neural effects of task order control and task set maintenance during dual-task processing. J Cogn Neurosci. 2008 Apr;20(4):613-28.

18. Stock A-K, Gohil K, Huster RJ, Beste C. On the effects of multimodal information integration in multitasking. Scientific Reports. 2017 Jul 7;7(1):4927. 
19. Szameitat AJ, Lepsien J, von Cramon DY, Sterr A, Schubert T. Task-order coordination in dualtask performance and the lateral prefrontal cortex: an event-related fMRI study. Psychol Res. 2006 Nov;70(6):541-52.

20. Yildiz A, Beste C. Parallel and serial processing in dual-tasking differentially involves mechanisms in the striatum and the lateral prefrontal cortex. Brain Struct Funct. 2014 Jul 14;112.

21. Downer MB, Kirkland MC, Wallack EM, Ploughman M. Walking impairs cognitive performance among people with multiple sclerosis but not controls. Human Movement Science. 2016 Oct 1;49(Supplement C):124-31.

22. Hamilton F, Rochester L, Paul L, Rafferty D, O'Leary C, Evans J. Walking and talking: an investigation of cognitive-motor dual tasking in multiple sclerosis. Mult Scler. 2009 Oct $1 ; 15(10): 1215-27$.

23. Holtzer R, Mahoney J, Verghese J. Intraindividual Variability in Executive Functions but Not Speed of Processing or Conflict Resolution Predicts Performance Differences in Gait Speed in Older Adults. J Gerontol A Biol Sci Med Sci. 2014 Aug;69(8):980-6.

24. Holtzer R, Wang C, Verghese J. Performance variance on walking while talking tasks: theory, findings, and clinical implications. AGE. 2014 Feb 1;36(1):373-81.

25. Learmonth YC, Ensari I, Motl RW. Cognitive Motor Interference in Multiple Sclerosis: Insights From a Systematic Quantitative Review. Archives of Physical Medicine and Rehabilitation. 2017 Jun 1;98(6):1229-40.

26. Wajda DA, Sosnoff JJ. Cognitive-Motor Interference in Multiple Sclerosis: A Systematic Review of Evidence, Correlates, and Consequences. Biomed Res Int [Internet]. 2015 [cited 2017 Nov 24];2015. Available from: https://www.ncbi.nlm.nih.gov/pmc/articles/PMC4369906/

27. Butchard-MacDonald E, Paul L, Evans JJ. Balancing the Demands of Two Tasks: An Investigation of Cognitive-Motor Dual-Tasking in Relapsing Remitting Multiple Sclerosis. Journal of the International Neuropsychological Society. 2017 ed;1-12.

28. Monticone M, Ambrosini E, Fiorentini R, Rocca B, Liquori V, Pedrocchi A, et al. Reliability of spatial-temporal gait parameters during dual-task interference in people with multiple sclerosis. A cross-sectional study. Gait \& Posture. 2014 Sep 1;40(4):715-8.

29. Beste C, Mückschel M, Paucke M, Ziemssen T. Dual-Tasking in Multiple Sclerosis - Implications for a Cognitive Screening Instrument. Front Hum Neurosci. 2018;12:24.

30. Welford AT. The 'Psychological Refractory Period' and the Timing of High-Speed Performance-a Review and a Theory. British Journal of Psychology General Section. 1952 Feb 1;43(1):2-19.

31. Pashler H. Processing stages in overlapping tasks: Evidence for a central bottleneck. Journal of Experimental Psychology: Human Perception and Performance. 1984 Jun;10(3):358-77.

32. Pashler H. Dual-task interference in simple tasks: data and theory. Psychol Bull. 1994 Sep;116(2):220-44. 
33. Wu C, Liu Y. Queuing network modeling of the psychological refractory period (PRP). Psychol Rev. 2008 Oct;115(4):913-54.

34. Dell'Acqua R, Pashler H, Stablum F. Multitasking costs in close-head injury patients. Exp Brain Res. 2003 Sep 1;152(1):29-41.

35. Van Selst M, Ruthruff E, Johnston JC. Can practice eliminate the Psychological Refractory Period effect? Journal of Experimental Psychology: Human Perception and Performance. 1999 Oct;25(5):1268-83.

36. Van Selst M, Jolicoeur P. Decision and Response in Dual-Task Interference. Cognitive Psychology. 1997 Aug 1;33(3):266-307.

37. Voigt I, Benedict M, Susky M, Scheplitz T, Frankowitz S, Kern R, et al. A Digital Patient Portal for Patients With Multiple Sclerosis. Front Neurol. 2020 May 22;11:400.

38. Rudick RA, Miller D, Bethoux F, Rao SM, Lee J-C, Stough D, et al. The Multiple Sclerosis Performance Test (MSPT): An iPad-Based Disability Assessment Tool. J Vis Exp [Internet]. 2014 Jun 30 [cited 2020 Mar 2];(88). Available from: https://www.ncbi.nlm.nih.gov/pmc/articles/PMC4209820/

39. Cella D, Lai J-S, Nowinski CJ, Victorson D, Peterman A, Miller D, et al. Neuro-QOL. Neurology. 2012 Jun 5;78(23):1860-7.

40. Bermel R, Mowry EM, Krupp L, Jones S, Naismith R, Boster A, et al. The Multiple Sclerosis Partners Advancing Technology and Health Solutions (MS PATHS) patient cohort (P4.381). Neurology. 2018 Apr 10;90(15 Supplement):P4.381.

41. Kurtzke JF. Rating neurologic impairment in multiple sclerosis. Neurology. 1983 Nov 1;33(11):1444.

42. Roxburgh RHSR, Seaman SR, Masterman T, Hensiek AE, Sawcer SJ, Vukusic S, et al. Multiple Sclerosis Severity Score. Neurology. 2005 Apr 12;64(7):1144.

43. Beste C, Yildiz A, Meissner TW, Wolf OT. Stress improves task processing efficiency in dualtasks. Behavioural Brain Research. 2013 Sep 1;252:260-5.

44. Manjaly Z-M, Harrison NA, Critchley HD, Do CT, Stefanics G, Wenderoth N, et al. Pathophysiological and cognitive mechanisms of fatigue in multiple sclerosis. J Neurol Neurosurg Psychiatry. 2019;90(6):642-51.

45. Hanken $\mathrm{K}$, Eling $\mathrm{P}$, Hildebrandt $\mathrm{H}$. Is there a cognitive signature for MS-related fatigue? Mult Scler. 2015 Apr;21(4):376-81.

46. Haase R, Schultheiss T, Kempcke R, Thomas K, Ziemssen T. Use and Acceptance of Electronic Communication by Patients With Multiple Sclerosis: A Multicenter Questionnaire Study. J Med Internet Res. 2012 Oct 15;14(5):e135.

47. Wojcik CM, Beier M, Costello K, DeLuca J, Feinstein A, Goverover Y, et al. Computerized neuropsychological assessment devices in multiple sclerosis: A systematic review. Mult Scler. 2019 Dec;25(14):1848-69. 
48. Mückschel M, Beste C, Ziemssen T. Immunomodulatory treatments and cognition in MS. Acta Neurol Scand. 2016 Sep;134 Suppl 200:55-9. 